\title{
Joint Model Predictive Control of Electric and Heating Resources in a Smart Building
}

\author{
Francesco Liberati Member, IEEE, Alessandro Di Giorgio Member, IEEE, Alessandro Giuseppi Student \\ Member, IEEE, Antonio Pietrabissa Member, IEEE, Emanuele Habib, and Luigi Martirano Senior Member, IEEE
}

\begin{abstract}
The new challenge in power systems design and operation is to organize and control smart micro grids supplying aggregation of users and special loads as electric vehicles charging stations. The presence of renewable and storage can help the optimal operation only if a good control manages all the elements of the grid. New models of green buildings and energy communities are proposed. For a real application they need an appropriate and advanced power system equipped with a building automation control system. This paper presents an economic model predictive control approach to the problem of managing in a coordinated way the electric and heating resources in a smart building, for the purpose of achieving in real time nearly zero energy consumption and automated participation to demand response programs. The proposed control, leveraging a mixed integer quadratic programming problem, allows to meet manifold thermal and electric users' requirements and react to inbound demand response signals, while still guaranteeing stable operation of the building's electric and thermal storage equipment. The simulation results, performed for a real case study in Italy, highlight the peculiarities of the proposed approach in the joint handling of electric and thermal building flexibility.
\end{abstract}

Index Terms-Economic Model Predictive Control, Heating Systems, Demand Side Management, Smart Buildings.

\section{NOMENCLATURE}

Indices

$e \quad$ Index of the generic electric vehicle $\mathrm{EV}$

$i \quad$ Time index in the MPC window

$k \quad$ Absolute time index

$l \quad$ Index of the generic plannable load

$u \quad$ Index of the generic housing unit

\section{Parameters}

$\gamma \quad$ Heat pump coefficient of performance

$\mathcal{T} \quad$ Sampling period

$\rho_{\text {air }} \quad$ Air density

$\xi^{e s s}$ Loss factor of the ESS battery pack

$\xi_{e}^{e s s} \quad$ Loss factor of the eth EV battery pack

$C^{\text {floor }}, C^{\text {wall }}$ Heat capacity of the floor and walls

$C_{i} \quad$ Electricity tariff at time $i$

$c_{i} \quad$ Cost of energy consumption at time $i$

$c_{a i r}, c_{w}$ Specific heat of the air and water

$E^{e s s} \quad$ Capacity of the ESS

$E_{e}^{e v} \quad$ Energy capacity of the $e$ th EV

$[\mathrm{kWh}]$

F. Liberati, A. Di Giorgio, A. Giuseppi and A. Pietrabissa are with the Department of Computer, Control, and Management Engineering Antonio Ruberti at Sapienza University of Rome, Via Ariosto, 25, 00185 Rome, Italy, e-mail: \{liberati,digiorgio,giuseppi,pietrabissa\}@diag.uniroma1.it.

E. Habib and L. Martirano are with the Department of Astronautics, Electrical and Energy Engineering of the Sapienza University of Rome, Via Eudossiana, 18, 00184 Rome, Italy, e-mail: \{emanuele.habib,luigi.martirano\}@uniroma1.it.
$F_{e} \quad$ End time of recharging session of EV $e$

$F_{l} \quad$ Latest allowed finish time of appliance $l$

$L_{i} \quad$ Set of plannable loads active at time $i$

$M \quad$ Boiler water mass

$N \quad$ Length of the MPC time window

$N_{l} \quad$ Duration of appliance program $l$

$P_{e}^{e v, \max }$ Maximum charging/discharging power of EV $e[\mathrm{~kW}]$

$P_{i}^{n p l} \quad$ Non plannable loads power absorption at time $i[\mathrm{~kW}]$

$P_{i}^{p l} \quad$ Power absorbed by the plannable loads at time $i[\mathrm{~kW}]$

$P_{i}^{p v} \quad$ Power produced by the PV panel at time $i \quad[\mathrm{~kW}]$

$R_{\text {floor }}^{a i r}$ Thermal resistance of the floor $\quad[\mathrm{K} / \mathrm{kW}]$

$R_{\text {wall }}^{\text {ext }}$ Thermal resistance of the external walls $\quad[\mathrm{K} / \mathrm{kW}]$

$R_{\text {floor }}^{\text {pipes }}$ Thermal resistance between the floor and the pipes

$[\mathrm{K} / \mathrm{kW}]$

$R_{\text {air }}^{\text {walls }}$ Thermal resistance of the walls $\quad[\mathrm{K} / \mathrm{kW}]$

$S_{e} \quad$ Start time of recharging session of EV $e$

$S_{l} \quad$ Earliest allowed start time for the program of appliance $l$ th

$U \quad$ Set of building's units

$V_{u} \quad$ Air volume in building's unit $u \quad\left[\mathrm{~m}^{3}\right]$ Variables

$S \tilde{O} C_{i}^{e s s}$ Reference state of charge of the ESS at time $i$ [\%]

$\tilde{T}_{u, i}^{a i r} \quad$ Reference air temperature in unit $u$ at time $i \quad\left[{ }^{\circ} \mathrm{C}\right]$

$\tilde{T}_{i}^{\text {boiler }}$ Reference boiler water temperature at time $i \quad\left[{ }^{\circ} \mathrm{C}\right]$

$P_{i}^{\text {ess }} \quad$ Power injected or absorbed by the ESS at time $i[\mathrm{~kW}]$

$P_{i}^{e v} \quad$ Aggregated power injected/absorbed by EVs at $i[\mathrm{~kW}]$

$P_{i}^{\text {poc }} \quad$ Power flow at the grid point of connection at $i[\mathrm{~kW}]$

$S O C_{i}^{e s s}$ State of charge of the ESS at time $i \quad[\%]$

$T_{u, i}^{a i r} \quad$ Air temperature in unit $u$ at time $i \quad\left[{ }^{\circ} \mathrm{C}\right]$

$T_{i}^{\text {boiler }}$ Boiler water temperature at time $i \quad\left[{ }^{\circ} \mathrm{C}\right]$

$T_{u, i}^{\text {pipes }}, T_{u, i}^{\text {floor }}, T_{u, i}^{\text {walls }}$ Pipes, floor and Walls temperature in

$\begin{array}{ll}\text { unit } u \text { at time } i & {\left[{ }^{\circ} \mathrm{C}\right]}\end{array}$

$\begin{array}{lll}T_{i}^{\text {ext }} & \text { External air temperature at time } i & {\left[{ }^{\circ} \mathrm{C}\right]}\end{array}$

Control Variables

$c_{i}^{\text {ess }} \quad c_{i}^{e s s} \in\{0,1\}, c_{i}^{e s s}=1$ if the ESS recharges at $i$

$c_{e, i}^{e v} \quad c_{e, i}^{e v} \in\{0,1\}, c_{e, i}^{e v}=1$ if EV $e$ recharges at $i$

$d_{i}^{\text {ess }} \quad d_{i}^{\text {ess }} \in\{0,1\}, d_{i}^{\text {ess }}=1$ if the ESS discharges at $i$

$d_{i, e}^{e v} \quad d_{i, e}^{e v} \in\{0,1\}, d_{i, e}^{e v}=1$ if EV $e$ discharges at $i$

$G_{u, i} \quad$ Water mass flow intake of housing unit $u$ at $i \quad[\mathrm{~kg} / \mathrm{s}]$

$P_{i}^{e s s, c}, P_{i}^{e s s, d}$ Charging/discharging ESS power at $i \quad[\mathrm{~kW}]$

$P_{e, i}^{e v, c}, P_{e, i}^{e v, d}$ Charging/discharging power of EV $e$ at $i \quad[\mathrm{~kW}]$

$P_{i, e}^{e v} \quad$ Power injected or absorbed by the EV $e$ at $i \quad[\mathrm{~kW}]$

$P_{i}^{h p} \quad$ Power absorbed by the heat pump at time $i \quad$ [kW]

$s_{l, j} \quad s_{l, j} \in\{0,1\}, s_{l, j}=1$ if the plannable program $l$ is set to start at time $j$ 


\section{INTRODUCTION}

\section{A. Motivation}

B UILDINGS and the construction sector are responsible, according to [1], for $30 \%$ of the final energy consumption, for more than $55 \%$ of the global electricity demand and for around $40 \%$ of the $\mathrm{CO}_{2}$ emissions. Increasing efficiency and smartness of the building stock is therefore fundamental to reduce greenhouse gas emissions, energy consumption and increase the share of Renewable Energy Sources (RES).

In Europe, the Energy Performance of Buildings Directive (2010/31/EU) [2], as amended in 2018 by the Directive (EU) 2018/844 [3], has established key principles, measures and binding targets, such as the introduction of minimum energy performance requirements for buildings, energy certification, mandates for nearly zero-energy performance in new buildings, for the installation of recharging points for Plug-in Electric Vehicles (PEVs), etc. The Directive also envisages separate regulation of the temperature in each room, vehicle to grid, deployment of high-capacity communication networks, building automation and control system to "support energy efficient, economical and safe operation of technical building systems through automatic controls..." [3] and introduces "the smart readiness indicator" for buildings, which "should be used to measure the capacity of buildings to use information and communication technologies and electronic systems to adapt the operation of buildings to the needs of the occupants and the grid and to improve the energy efficiency and overall performance of buildings" [3]. The aggregation of small and medium users in a cluster or community is one of the most exciting challenges in the field of power systems, in which the building automation and the controls play a fundamental role.

\section{B. Purpose of the paper}

This paper proposes a Model Predictive Control (MPC) approach to the problem of managing, in a coordinated and synergic way, the electric and heating resources in a smart building, for the purpose of achieving, in real time, nearly zero energy consumption and automated participation to Demand Response (DR) programs, while satisfying users comfort. The reference scenario (Fig. 1) considers a mixed-use building consisting of several units (e.g. shops, common areas, residential units, etc.), equipped with centralized local energy resources, such as: photovoltaic (PV) panels, electric storage system (ESS), heating system, ground-source heat pump, and individual electricity smart (controllable/plannable) loads such as appliances and PEVs charging stations. A centralized hot water storage provides heating to each unit and can be used as a controllable thermal storage.

The controllable variables of the system (i.e., the variables manipulated in order to control the behavior of the system) include the power absorption of the heat pump and the opening of the hot water flow valves at the single building units (which affect both the temperature of the water in the boiler and the air temperature in the single units of the building), the start times of smart appliances, the charging and discharging power of the ESS and the PEVs (which, together with the heat pump control and the local power generation, affect the overall net

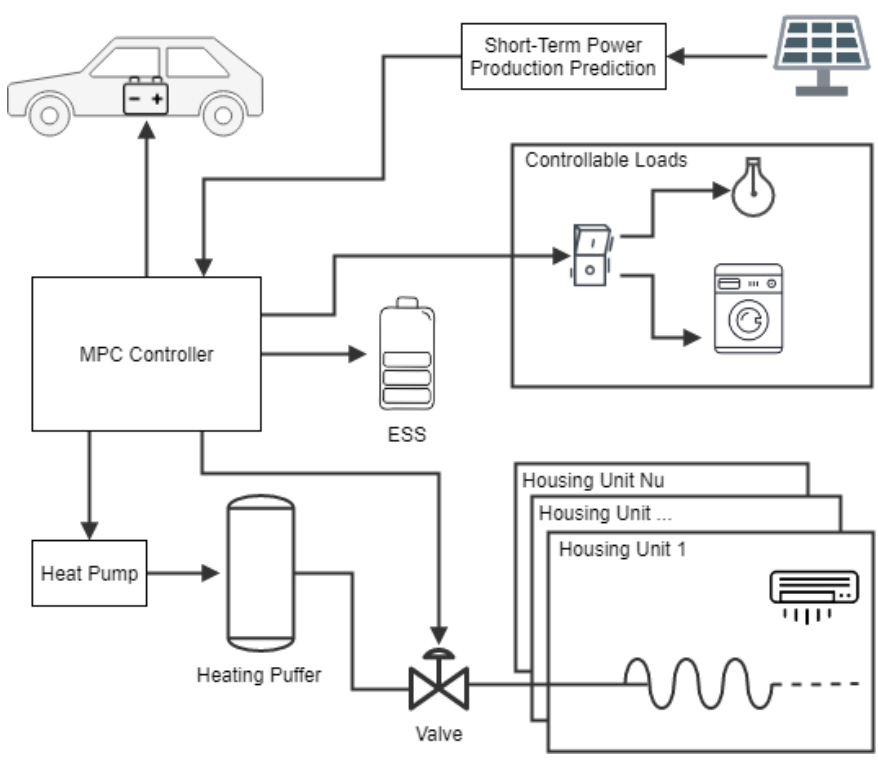

Fig. 1. Reference architecture of a smart building, consisting of several units heated by a centralized controlled system.

power withdrawal of the building). The controlled variables of the system are the temperature of the hot water storage, the air temperature in the building's units, the State of Charge (SOC) of the ESS, the PEVs' SOC and the overall building net power withdrawn from the grid. More in particular, the control objectives include: 1) to ensure comfort of the building occupants, in particular, to ensure that the set temperature in each unit (possibly different from unit to unit) is tracked when the inhabitant desires; 2) to minimize the electricity bill of the building, also in presence of complex electricity tariff models; 3) to minimize the power flow at the point of coupling of the building with the grid; 4) more in general, to be able to control the power flow at the point of connection with the grid, thus enabling the building e.g. to participate in DR programs; 5) to control the ESS SOC and the temperature of the hot water storage (which, together with the air temperature in the units, are the state variables of the overall controlled system), so that they remain close to an appropriate reference value to guarantee sufficient upside and downside control margins for the system. The overall control problem formulation includes terms accounting for all of the above objectives, so that the resulting system will be able to work in all of the use cases resulting from prioritizing one or more of the control objectives over the remaining ones. The proposed problem falls into the category of Economic Model Predictive Control (EMPC), which is an optimization-based control technique in which the objective function has an economic or general performance based meaning (rather than a stabilizing meaning, as in classical MPC [4]). The objective function is quadratic (to penalize deviations of the controlled temperatures and SOC from the reference setpoints) and includes mixed-integer terms, also to be able to model and correctly price power flows from/to the grid. Constraints include user comfort and systems' technical constraints, and the dynamics equations of the system, which model the evolution of air temperature in 
the building's units, water temperature in the boiler, and SOC of the ESS and PEVs.

A real case study of a building with a micro grid in Italy is taken into account for the modelling, dimensioning and calibration of the system's equations. Simulation results are proposed to show how the performance of the building (in terms of the control objectives proposed above) can be optimized by leveraging synergies of the heat and electric resources available, by maximizing self-consumption of locally generated power, (heat and electric) storage (also including buildings thermal inertia), modulation and shifting of loads to exploit tariff valleys, etc.

\section{Structure of the paper}

The remainder of the paper is organised as follows. Section II discusses the state of the art, providing an overview about the factors influencing the design of Energy Management Systems (EMSs) in buildings and the main approaches used to address the electric and thermal load management problem. Section III presents the control problem formalization. Section IV presents the case study and reports the simulation results. Finally, Section V concludes the work.

\section{STATE OF THE ART AND PROPOSED CONTRIBUTION}

In recent years, the topic of load control as a mean for enabling smart households and buildings to nearly zero energy consumption and DR functionalities has been the subject of intense research. Reviews of the many concepts and methods in EMSs design and DR problems can be found, e.g., in [5], [6] and [7]. For the sake of what follows, it is instrumental to remark that the load control problem is influenced by several factors, such as the type of loads [8] and generation [9], the pricing scheme [10], the occupancy of the household [11] and the weather [12]. All these factors result in a variability of performances, as testified by a variety of pilot projects running in Germany [13], Belgium [14], the Netherlands [15], etc.

As a matter of fact, the use of an EMS for automated participation in DR programs is largely recognized as fundamental in challenging scenarios, in which multiple sources of energy like local generators from RES and an ESS have to be managed, in combination with several classes of loads, such as smart appliances, PEVs, and in presence of complex electricity price structures such as Time Of Use (TOU), Day Ahead Pricing (DAP), Critical Peak Pricing (CPP), Real Time Pricing (RTP) [16], Multi-TOU and Multi-CPP [17]; such pricing schemes are introduced by retailers to achieve responsiveness of users and share the risk of market volatility. Furthermore, the problem complexity is emphasized by the need of considering the heating system as part of the problem, in order to achieve control on the overall building energy consumption.

Rule-based methods are widely used in practice in current EMSs because of their simplicity and low computation effort [18]. Though easy to implement, these methods may be oversimplified and do not allow to capture the near future behaviour of the process under control. Advanced EMSs typically rely on different forms of optimization, such as linear programming [19], binary linear programming [20], nonlinear programming [21], Mixed Integer Linear Programming (MILP) [22], [23], [24], mixed-integer nonlinear programming [25] and multiparametric programming [26]. A detailed description of the above mentioned papers can be found in [27]. In particular MILP formulations are useful not only to manage loads characterized by different levels of controllability (on/off, continuous and semicontinuous controls), but also to handle nonlinearities introduced by the different prices that energy may have depending on its flow direction at the point of connection between the building and the grid [28]. In general, optimization is currently recognized to be effective when integrated in a MPC framework, due to its intrinsic ability to handle multivariable and non-stationary control problems, also in presence of inaccurate models.

When the optimization problem is too complex to be solved leveraging standard methods, techniques such as genetic algorithms [29], particle swarm optimization [30] and metaheuristic tabu search [31] are used. Other approaches that can be found applied in this research area are the Lagrangian relaxation [32] and Benders decomposition [33]. In-depth reviews about the used optimization techniques can be found in [34] and [35]. Finally, also learning techniques have been applied in the context of residential EMSs, such as artificial neural networks [36] and reinforcement learning applied to Markov decision processes [37]. Interested readers may refer to [7] for an insight on artificial intelligence based EMSs.

Though the building heating system is commonly recognized as a potential source of flexibility, most of the related articles in literature have their special focuses with limited attention on the interactions among thermal equipment/requirements and other controllable electric loads for the purpose of overall nearly zero energy consumption and DR.

Some papers are available on the partial integration of controllable appliances and Heating, Ventilation and Air Conditioning (HVAC) systems, which mainly focus on Combined Heat and Power (CHP) management [38], [39], [40] and [41]. In [42] an optimization based strategy is proposed for integrating variable wind power with the CHP, while still satisfying customers heating demand changes. In [43] it is shown how aggregates of thermostatically controlled loads (refrigerators, air-conditioners and space heaters) offer significant potential for the provisioning of frequency response services.

In [44], an EMPC strategy is proposed to shift the heating energy consumption according to the time varying energy price. The investigated heating system consists of a heat pump integrated with a hot water tank as active thermal energy storage; two optimization problems are integrated together to optimize both the heat pump electricity consumption and the building heating consumption. In [45], a similar problem is faced, with a focus on the use of price signals to defer the heating loads so that the profit of the retailer is maximized. Finally, in [46] a similar MPC strategy is proposed for the integration of thermal energy storage, HVAC and local RES. The last three papers, though taking into account limited scenarios in terms of managed equipment and resources, incorporate in a basic form some concepts which are at the basis of the present work, like the use of thermal flexibility 
as a mean for shaping the net building electricity withdrawal, optimally exploiting local RES and providing power balancing services to market players.

In the present work, the performance of the building is optimized by leveraging synergies of the heat and electric resources. The main contribution of the paper and consequent advancement beyond the state of the art is given by the completeness and detail of the scenario investigated (in terms of controlled equipment, control objectives and business cases enabled) and the proposition of a particularly advanced and again complete mathematical problem formulation in addressing combined and synergic management of heat and electric resources. The paper achieves this by combining and extending two lines of research from the authors, one focused mainly on building thermal management [47], and the second, developed in the series of works [20],[22],[27] dedicated to the control of electric building resources for the purpose of automated participation to DR programs. The analysis, and consequent design, of the MPC controller presented in [47] was tailored by the authors specifically for the heating system control of a Nearly Zero Energy Building (NZEB), without considerations on potential synergies with power component of the building. This integration required a re-modeling of the thermal dynamics and considerations of the system (as described by (21)-(28) below), to cope with the increased complexity bought by the MILP formulation of the electrical subsystem. Regarding DR, a first contribution was given by the authors in [48] and [49], where the aggregation of housing units of a NZEB is envisaged for the participation of the building to DR programs. In those papers, the authors dealt with the control issue empirically, with discrete actions on the setpoints of the local controllers. The present paper, combining the complex specifics of the proposed load controllers, as [27], to EMSs interfaced with HVAC units, and PEV charging stations, deals with a different approach to the combined control of electric and thermal loads, based on MPC, which enables integrated scenarios characterized by significant control flexibility and exploitation of the integration into the problem formulation of the system dynamics models.

Finally, the present paper also includes, in Section III.D, a discussion of the stability properties of the control system, something that is rarely found in literature for such rich problem formulations deriving from real application requirements.

\section{MATHEMATICAL FORMULATION}

\section{A. Objective function}

As customary in EMPC, we consider a multi-objective function that captures the physical behaviour of the system and also its economical performance:

$$
\begin{aligned}
V= & \sum_{i=k}^{k+N-1}\left\{a_{1} c_{i}+a_{2} \sum_{u \in U}\left(T_{u, i}^{a i r}-\tilde{T}_{u, i}^{a i r}\right)^{2}+\right. \\
& +a_{3}\left(T_{i}^{\text {boiler }}-\tilde{T}_{i}^{\text {boiler }}\right)^{2}+ \\
& \left.+a_{4}\left(S O C_{i}^{\text {ess }}-S \tilde{O} C_{i}^{e s s}\right)^{2}+a_{5}\left(P_{i}^{\text {poc }}\right)^{2}\right\}
\end{aligned}
$$

in which $k$ is the current time and $\left\{a_{i}\right\}_{i=1, \ldots, 5}$ are the weights of the different terms, dictating their relative importance in the multi-objective problem. The summation over $N$ steps into the future represents the so-called "prediction horizon" of the EMPC, since it allows the controller to take actions accounting also for the predicted behaviour of the system. The predictions needed for the evaluation of a cost function with the structure of (1) are obtained thanks to the model of the system that is embedded in the controller itself and, for exogenous signals, from short-term predictions. Parameter $N$, the length of the prediction horizon, is tunable. The longer the prediction horizon, the higher is the complexity of the problem (the higher the number of variables of the problem). In a nominal setting (without any uncertainty), a longer prediction horizon brings the performance of the MPC algorithm closer to the optimum achievable value (i.e. the solution of an infinitehorizon open loop optimization problem). The adoption of a large $N$ is however not advisable because of the uncertainty affecting the forecasts. Given the dynamics of the systems at study, a reasonable value for $N$ in our view is from some minutes to less than 24 hours (we chose $N=3$ hours, which results in fast solving time and good performance).

EMPC follows the receding horizon paradigm introduced for MPC, as the open-loop problem of the minimization of (1) is repeated at every time step $k$, consequently applying to the system only the first element of the control sequence that optimized the objective function. At each iteration the optimization is done after having observed the system state, hence compensating any discrepancies between the predicted evolution of the system and its real behaviour. This combination of optimization and the iterative state feedback is what gives MPC/EMPC high performance and robustness.

We now briefly discuss (1). The first term accounts for the minimization of the overall electricity bill of the building, and is defined further below. The second term is related to users' comfort and penalizes the deviations of the air temperature in each unit from a given set-point. Air temperature setpoints are typically preset by the building manager; they are usually time-varying and they can be changed by the users via the thermostats in the units. The third and the fourth terms penalize, respectively, the deviations of the boiler water temperature and of the ESS SOC from given set-points. They are included to keep the boiler and the ESS close to a state in which they can exchange significant amounts of heat and electric energy (i.e. so to have significant upward and downward control margins). A reasonable choice for $\tilde{T}_{i}^{\text {boiler }}$ and $S \tilde{O} C_{i}^{e s s}$ can be therefore $50 \%$ of the allowed temperature and SOC range. The last term penalizes high electric power flows at the point of connection with the grid.

\section{B. Constraints}

One of the main advantages of EMPC is its ability to handle constraints with ease, thanks to the receding horizon paradigm that leads to subsequent optimizations over a limited prediction horizon. Constraints below hold for all $i \in[k, k+N-1]$, $u \in U, e \in E$ (the sets of building units and PEVs controlled).

Definition of $c_{i}$ : The term $c_{i}$ in (1) is the cost/profit deriving from the energy absorbed or injected into the grid at time $i$. It is a nonlinear term because, to make the formulation of 
the problem more general and aligned with the reality, it is assumed here that the energy tariff depends on the sign of $P_{i}^{p o c}$. That is, the cost for buying energy (i.e. when $P_{i}^{p o c} \geq 0$ ) is assumed different in general from the remuneration received when power is injected into the grid (i.e. when $P_{i}^{p o c} \leq 0$ ). Let us then denote the tariff at $i$ with $C_{i}\left(P_{i}^{p o c}\right)$, to make explicit its dependence on $P_{i}^{p o c}$. Given this additional notation, it is $c_{i}=$ $\mathcal{T} P_{i}^{p o c} C_{i}\left(P_{i}^{p o c}\right)$. The term can be replaced with an equivalent linear one adapting the approach explained in [27]. Boolean variables $\delta_{i}^{1}$ and $\delta_{i}^{2}$ are added, with the following constraints:

$$
\begin{gathered}
\delta_{i}^{1}+\delta_{i}^{2}=1 \\
P^{p o c, \min } \delta_{i}^{1} \leq P_{i}^{\text {poc }} \leq P^{\text {poc, } \max } \delta_{i}^{2}
\end{gathered}
$$

It can be verified that, with the above positions, it is $\delta_{i}^{1}=1$ if and only if $P_{i}^{p o c} \leq 0$ and $\delta_{i}^{2}=1$ if and only if $P_{i}^{\text {poc }} \geq 0$. Next, let us further denote with $c_{i}^{1}$ the remuneration price obtained at $i$ when power is injected into the grid (i.e. when $\left.\delta_{i}^{1}=1, P_{i}^{p o c} \leq 0\right)$ and with $c_{i}^{2}$ the energy cost when power is consumed from the grid (i.e. when $\delta_{i}^{2}=1, P_{i}^{\text {poc }} \geq 0$ ). With the above definitions and constraints, the cost at $i$ can be written as $c_{i}=\mathcal{T} P_{i}^{\text {poc }}\left(\delta_{i}^{1} c_{i}^{1}+\delta_{i}^{2} c_{i}^{2}\right)$. It is only left to transform the products $P_{i}^{\text {poc }} \delta_{i}^{1}$ and $P_{i}^{\text {poc }} \delta_{i}^{2}$ into a linear expression, which can be done by adding two additional real valued variables, $z_{i}^{1}$ and $z_{i}^{2}$, together with the following constraints:

$$
\begin{aligned}
& P^{\text {poc }, \min } \delta_{i}^{1} \leq z_{i}^{1} \leq P^{\text {poc, } \max } \delta_{i}^{1} \\
& P^{\text {poc,min }} \delta_{i}^{2} \leq z_{i}^{2} \leq P^{\text {poc, } \max } \delta_{i}^{2} \\
& P_{i}^{\text {poc }}-\left(1-\delta_{i}^{1}\right) P^{p o c, \max } \leq z_{i}^{1} \leq P_{i}^{\text {poc }}-\left(1-\delta_{i}^{1}\right) P^{\text {poc,min }} \\
& P_{i}^{\text {poc }}-\left(1-\delta_{i}^{2}\right) P^{p o c, \max } \leq z_{i}^{2} \leq P_{i}^{\text {poc }}-\left(1-\delta_{i}^{2}\right) P^{\text {poc,min }}
\end{aligned}
$$

It can be verified that, with the above additional constraints, it is $z_{i}^{1}=P_{i}^{p o c} \delta_{i}^{1}$ and $z_{i}^{2}=P_{i}^{p o c} \delta_{i}^{2}$. Hence, finally, the cost term $c_{i}$ can be rewritten in linear form as:

$$
c_{i}=\left(c_{i}^{1} z_{i}^{1}+c_{i}^{2} z_{i}^{2}\right) \mathcal{T}
$$

Definition of $P_{i}^{p o c}$ : The electric power flow is defined by the following power balance equation.

$$
P_{i}^{p o c}=P_{i}^{h p}+P_{i}^{e v}+P_{i}^{e s s}+P_{i}^{p l}+P_{i}^{n p l}+P_{i}^{p v},
$$

where, in particular:

- $P_{i}^{h p}$ is the power consumption of the heat pump;

- $P_{i}^{e v}$ (the aggregated power consumption of the PEVs) is:

$$
P_{i}^{e v}=\sum_{e \in E_{i}} P_{e, i}^{e v},
$$

where $E_{i}$ is the set of PEVs connected at time $i\left(E_{i}:=\right.$ $\left\{e \in E: S_{e} \leq k \leq F_{e}\right\}$ ) and $P_{e, i}^{e v}$ (the power from/to the single $\mathrm{PEV})$ is defined as in (5)-(8) in [27]:

$$
P_{e, i}^{e v}=P_{e, i}^{e v, c}+P_{e, i}^{e v, d}
$$

subject to

$$
\begin{gathered}
0 \leq P_{e, i}^{e v, c} \leq P_{e}^{e v, \max } c_{e, i}^{e v} \\
0 \leq-P_{e, i}^{e v, d} \leq-P_{e}^{e v, \min } d_{i, e}^{e v} \\
c_{e, i}^{e v}+d_{i, e}^{e v} \leq 1
\end{gathered}
$$

where $P_{e, i}^{e v, c}$ and $P_{e, i}^{e v, d}$ are, respectively, the charging and discharging power, $P^{e v, \max }$ the maximum charging/discharging power, $c_{e, i}^{e v}$ a charging indicator variable (equal to 1 if $\mathrm{PEV} e$ is charging at time $i$ ) and $d_{i, e}^{e v}$ the Boolean discharging indicator variable;

- $P_{i}^{e s s}$ is similarly defined as

$$
P_{i}^{e s s}=P_{i}^{e s s, c}+P_{i}^{e s s, d}
$$

subject to

$$
\begin{aligned}
& 0 \leq P_{i}^{e s s, c} \leq P^{e s s, \max } c_{i}^{e s s} \\
& 0 \leq-P_{i}^{e s s, d} \leq-P^{e s s, m i n} d_{i}^{e s s} \\
& c_{i}^{e s s}+d_{i}^{e s s} \leq 1
\end{aligned}
$$

with similar variables and parameters defined;

- $P_{i}^{p l}$ (the aggregated consumption of plannable loads) is defined as in (3) in [27]:

$$
P_{i}^{p l}=\sum_{l \in L_{i}} \sum_{j=j_{l}^{1}}^{j_{l}^{2}} P_{l, i-j+1} s_{l, j},
$$

where $P_{i}^{p l}$ denotes the sum of the power consumed by the plannable loads at time $i$. Variable $s_{l, j}$ is a Boolean decision variable and it is $s_{l, j}=1$ if the plannable load $l$ is started at time $j . P_{i}^{p l}$ is hence derived by considering the set of loads $L_{i}$ potentially active at $i$ :

$$
L_{i}:=\left\{l \in L_{k}: S_{l} \leq i \leq \min \left(F_{l}, k+N-1\right)\right\}
$$

$L_{k}$ in turn denotes the set of loads to be managed at time $k$, i.e. the set of loads that have been requested by the users before $k$ and are not started yet:

$$
L_{k}:=\left\{l \in L:\left(R_{l} \leq k\right) \&\left(\text { state }_{l, k}=0\right)\right\}
$$

state $_{l, k}$ is the state of the load at $k$ : equal to 0 if the load has not started yet, equal to 1 otherwise if the load is already in execution at $k$.

The update equation for the generic load is:

$$
\text { state }_{l, i+1}=\left\{\begin{array}{lll}
1, & \text { if } & \text { state }_{l, i}=1 \\
1, & \text { if } & s_{l, k}^{*}=1 \\
0, & \text { if } & s_{l, k}^{*}=0
\end{array}\right.
$$

where $s_{l, k}^{*}$ denotes the optimal value of $s_{l, k}$.

Coming back to (15), for each load $i$, the exact power consumption of the load at time $i$ depends on the user preferences: $j_{l}^{1}$ denotes the earliest possible starting time such that the load will have power consumption at $i$, while $j_{l}^{2}$ is the last start time such that the load will contribute to power consumption at $i$. They are given by:

$$
\begin{gathered}
j_{l}^{1}=\max \left(\max \left(k, S_{l}\right), i-N_{l}+1\right) \\
j_{l}^{2}=\min \left(i, \min \left(F_{l}, k+N-1\right)-N_{l}+1\right) .
\end{gathered}
$$

Exactly one starting time is decided for each load:

$$
\sum_{i=S_{l}}^{F_{l}-N_{l}+1} s_{l, i}=1
$$


Dynamics of the boiler's water temperature (i.e. definition

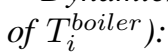

$$
\begin{aligned}
T_{i+1}^{\text {boiler }}= & T_{i}^{\text {boiler }}+\frac{\mathcal{T}}{M c_{w}}\left[\gamma P_{i}^{h p}+\right. \\
& \left.-\sum_{u \in U} G_{u, i} c_{w}\left(T_{i}^{\text {boiler }}-T_{u, i}^{\text {pipes }}\right)-\alpha T_{i}^{\text {boiler }}\right] \\
& T_{k}^{\text {boiler }}=T^{\text {boiler }, k},
\end{aligned}
$$

where $\gamma$ is the Coefficient of Performance (COP) of the heat pump, a positive value (larger than one) that captures the power required to move heat from an energy source (e.g., water, air, ground) to the boiler. The rest of the equation is derived from a simple energy balance, as the control variable $G_{u, i}$, the heated water mass flow governed by the valve that regulates the heating of the housing unit $u$, governs the flow that transfers heat from the boiler to the housing unit $u$. The sum of all of these heat flows represents the total heat demand that the boiler should satisfy. The last term in (21) represents the heat loss of the boiler, governed by the factor $\alpha \geq 0$.

Due to the presence of the product between a control variable, $G_{u, i}$, and state variables, considering equation (21) as a constraint for our problem would cause a significant increase in computational complexity. For this reason, we transform (21) into a liner equivalent with the following substitution:

$$
\sum_{u \in U} G_{u, i} c_{w}\left(T_{i}^{\text {boiler }}-T_{u, i}^{\text {pipes }}\right)=\sum_{u \in U} \frac{\left(T_{u, i}^{\text {pipes }}-T_{u, i}^{\text {floor }}\right)}{R_{\text {floor }}^{\text {pipes }}} .
$$

The right hand side of (22) represents the heat exchanged between the heating pipes and the floors of the various housing units, utilizing the concept of thermal resistance. Under the assumption that no heat is lost in the distribution system, the two sides of (22) represent the same quantity, i.e., the heat flow that the boiler provides, overall, to the housing units. Equation (21) then becomes

$$
\begin{aligned}
T_{i+1}^{\text {boiler }}= & T_{i}^{\text {boiler }}+\frac{\mathcal{T}}{M c_{w}}\left[\gamma P_{i}^{\text {hp }}+\right. \\
& \left.-\sum_{u \in U} \frac{\left(T_{u, i}^{\text {pipes }}-T_{u, i}^{\text {floor }}\right)}{R_{\text {floor }}^{\text {pipes }}}-\alpha T_{i}^{\text {boiler }}\right] \\
& T_{k}^{\text {boiler }}=T^{\text {boiler }, k} .
\end{aligned}
$$

Due to substitution (22), the control variable in the equation is no longer $G_{u, i}$, but $T_{u, i}^{\text {pipes }}$ instead. This change of variable is legitimate, as we can show that, under reasonable assumptions, there exists an invertible relation between the two.

Assuming a linear relation between the opening of the control valve and the mass flow $G_{u, i}$ through it, as well as ideal mixing condition in the pipes, we can write

$$
T_{u, i}^{\text {pipes }}=T_{u, i}^{\text {floor }}+\frac{G_{u, i}}{G_{u}^{\text {max }}}\left(T_{i}^{\text {boiler }}-T_{u, i}^{\text {floor }}\right),
$$

with $G_{u}^{\max }$ the maximum mass flow that the valves allow, making the substitution possible from a mathematical point of view. Moreover, to reflect the physical limitation of the mass flow (i.e., $0 \leq G_{u, i} \leq G_{u}^{\max }$ ) on the new control variable, two constraint are added to specify that the temperature of water in the pipes: i) cannot exceed the temperature of the boiler (that is reached when the control valve is completely open) and ii) is at least at temperature equilibrium with the floor (the case when the control valve is completely closed):

$$
T_{u, i}^{\text {floor }} \leq T_{u, i}^{\text {pipes }} \leq T_{i}^{\text {boiler }} .
$$

We detail in the following the dynamics of the various temperatures of interest characterizing each building unit.

Dynamics of the units' floor temperature (i.e. definition of $T_{u, i}^{\text {floor }) \text { : }}$

$$
\begin{gathered}
T_{u, i+1}^{\text {floor }}=T_{u, i}^{\text {floor }}+\frac{\mathcal{T}}{C^{\text {floor }}}\left[\frac{T_{u, i}^{a i r}-T_{u, i}^{\text {floor }}}{R_{\text {floor }}^{\text {air }}}+\right. \\
\left.+\frac{T_{u, i}^{\text {pipes }}-T_{u, i}^{\text {floor }}}{R_{\text {floor }}^{\text {pipes }}}\right], \\
T_{u, k}^{\text {floor }}=T_{u}^{\text {floor }, k},
\end{gathered}
$$

Equation (26) describes the effects of the control variable $T_{i}^{\text {pipes }}$ on $T_{u, i+1}^{\text {floor }}$. The floor exchanges heat with both the pipes it contains and with the air of the unit. These exchanges are driven, respectively, by the temperature differences $T_{u, i}^{a i r}-$ $T_{u, i}^{\text {floor }}$ and $T_{u, i}^{\text {pipes }}-T_{u, i}^{\text {floor }}$. The thermal resistances $R_{\text {floor }}^{\text {air }}$

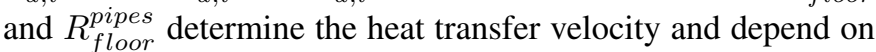
the materials (as all the other thermal resistances considered).

Dynamics of the units' air temperature (definition of $T_{u, i}^{a i r}$ ):

$$
\begin{gathered}
T_{u, i+1}^{a i r}=T_{u, i}^{a i r}+\frac{\mathcal{T}}{c_{a i r} \rho_{a i r} V_{u}}\left[\frac{T_{u, i}^{\text {floor }}-T_{u, i}^{a i r}}{R_{\text {floor }}^{\text {air }}}+\right. \\
\left.+\frac{T_{u, i}^{\text {walls }}-T_{u, i}^{\text {air }}}{R_{\text {air }}^{\text {walls }}}\right], \\
T_{u, k}^{a i r}=T_{u}^{a i r, k},
\end{gathered}
$$

where $c_{a i r}$ is the specific heat of the air and $\rho_{\text {air }}$ the density, while $V_{u}$ is the air volume in unit $u$. Equation (27) describes the heat exchanged between the air in the unit and the architectural elements. We did not include the effect of air ventilation and heat flows from powered appliances, due to their negligible effects on the heating system in winter days.

Dynamics of the units' walls temperature (definition of $T_{u, i}^{\text {walls }):}$

$T_{u, i+1}^{\text {walls }}=T_{u, i}^{\text {walls }}+\frac{\mathcal{T}}{C^{\text {wall }}}\left[\frac{T_{u, i}^{\text {air }}-T_{u, i}^{\text {walls }}}{R_{\text {air }}^{\text {walls }}}+\frac{T_{i}^{\text {ext }}-T_{u, i}^{\text {walls }}}{R_{\text {walls }}^{\text {ext }}}\right]$,

$T_{u, k}^{w a l l s}=T_{u}^{w a l l s, k}$

where $T_{i}^{e x t}$ is the external air temperature at time $i$, causing a heat loss for the building and a decrease in the ambient air temperatures, to be compensated by the heating controller.

Finally, operational constraints can be added, typically on the maximum or minimum allowed temperatures of operations, such as, in our case, $T_{i}^{\text {boiler }}>38{ }^{\circ} \mathrm{C}$ (to make sure there is always enough domestic hot water) and $T_{u, i}^{\text {floor }}<35^{\circ} \mathrm{C}$ (as maximum floor temperatures is limited by comfort issues). 
Dynamics of the ESS (i.e. definition of $S O C_{i}^{\text {ess }}$ ):

$$
\begin{aligned}
& S O C_{i+1}^{e s s}=S O C_{i}^{e s s}+\frac{\mathcal{T} \cdot\left(P_{i}^{e s s}-\xi^{e s s}\left|P_{i}^{e s s}\right|\right)}{E^{e s s}} 100, \\
& S O C_{k}^{e s s}=S O C^{e s s, k}
\end{aligned}
$$

The SOC is expressed in $\%$ of the full charge. The term $\xi^{e s s}\left|P_{i}^{e s s}\right|$ models the power losses associated with charging and discharging operation, and can be easily linearised considering that $\left|P_{i}^{\text {ess }}\right|=P_{i}^{\text {ess, }}+P_{i}^{\text {ess }, d}$.

Dynamics of the PEVs (i.e. definition of $S O C_{e, i}^{e v}$ ):

$$
\begin{aligned}
& S O C_{e, i+1}^{e v}=S O C_{e, i}^{e v}+\frac{\mathcal{T} \cdot\left(P_{e, i}^{e v}-\xi_{e}^{e v}\left|P_{e, i}^{e v}\right|\right)}{E_{e}^{e v}} 100, \\
& S O C_{e, k}^{e v}=S O C^{e v, e, k} \\
& S O C_{e, F_{e}}^{e v}=S O C^{e v, e, F_{e}}
\end{aligned}
$$

where $S O C^{e v, e, F_{e}}$ is the desired final SOC.

Variables range: The controlled variables must lie in a range between a minimum and a maximum allowed value.

$$
\begin{gathered}
T_{u, i}^{a i r, m i n} \leq T_{u, i}^{a i r} \leq T_{u, i}^{a i r, \text { max }} \\
T_{i}^{\text {boiler,min }} \leq T_{i}^{\text {boiler }} \leq T_{i}^{\text {bolier,max }} \\
S O C^{e s s, \text { min }} \leq S O C_{i}^{e s s} \leq S O C^{e s s, \text { max }} \\
S O C_{e}^{e v, \text { min }} \leq S O C_{e, i}^{e v} \leq S O C_{e}^{e v, \text { max }}
\end{gathered}
$$

Notice that the constraints on power and SOC cannot be physically violated if $\min$ and $\max$ are $0 \%$ and $100 \%$ of the allowed range. Instead the constraints on temperature can be physically violated (they are soft constraints).

Variables nature:

$$
\begin{gathered}
S O C^{e s s}, S O C^{\text {pev }}, P, P^{e s s} \in \mathbb{R} \\
P^{\text {pl }}, P^{\text {pev }}, P^{e s s, d}, P^{e s s, c}, P^{\text {pev }, d}, P^{\text {pev }, c} \in \mathbb{R} \\
T^{\text {boiler }}, T^{\text {pipes }}, T^{\text {floor }}, T^{\text {air }}, T^{\text {walls }} \in \mathbb{R} \\
s_{l}, c^{\text {ess }}, d^{e s s}, c^{\text {pev }} \in\{0,1\} \\
d^{\text {pev }}, \delta P_{j}, z_{j} \in\{0,1\}
\end{gathered}
$$

C. Summary of the inputs and outputs of the control system, and of the overall MPC iteration

The MPC control signals are summarized in the nomenclature section, under the label "control variables" and mainly consist in power profiles for the active elements of the system (ESS, PEVs, heat pump, plannable loads) and the actuation profile of the heating system valves. The inputs needed from the controller are also reported in the nomenclature, under the "variables" label. Inputs are mainly given by the feedback on the current state of the system and the reference signals (reference temperatures, reference ESS SOC). All the other symbols introduced in the previous sections are parameters and auxiliary variables needed to build the MPC model.

The MPC control algorithm can be summarized as follows. At each time $k, k+1, \ldots$ do:

1) Solve:

$$
\begin{aligned}
& \min (1) \\
& \text { subject to } \\
& (2)-(20),(23),(25)-(32)
\end{aligned}
$$

2) Apply to the system the first sample of the found optimal control variables.

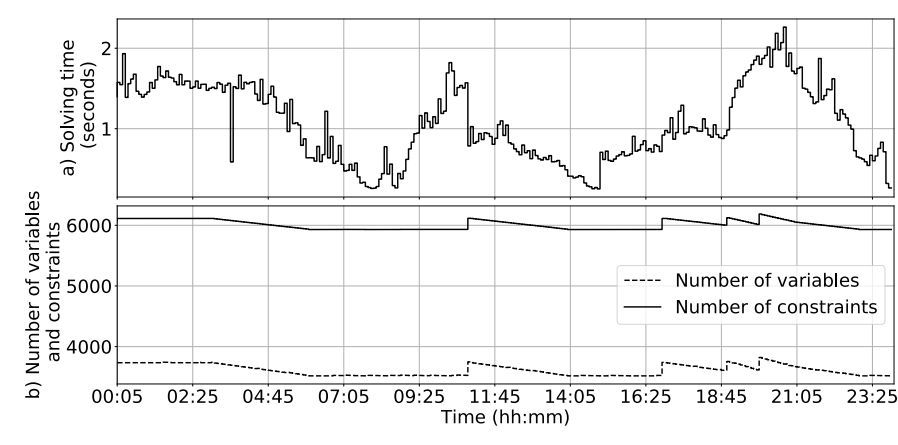

Fig. 2. Solving time (a) and number of variables and constraints for the optimization problem to be solved at each time (b) in Simulation 3.

\section{Problem Complexity, Feasibility and Stability}

1) Problem Complexity: Figure 2 reports, in the first subplot, the solving time of each of the MPC iterations done in Simulation 3 (the most complex one). The second subplot reports the total number of constraints and variables in each iteration. This gives an idea of the dimension of the MPC iterations and the time complexity. Time complexity is compatible with the real implementation of the controller (the solving time is well below the sampling time, 5 minutes).

2) Problem Feasibility: The constraints that can generate infeasibility are the ones reflecting real physical constraints or limitations existing in the controlled system, that is, (2) and (3) (limits of the power at the point of connection, $P^{p o c}$ ); (8) and (9) (PEV power charging limits); (12) and (13) (ESS power charging limits); the thermal constraints (23), (26), (27), (28), which all have a similar mathematical structure, (25) on the valve opening limits; (29) and (30) (on the SOC of the ESS and the PEV), and finally the variables' box constraints (31). The remaining constraints do not reflect actual limitations, but are instead needed to define auxiliary variables (see e.g. (4)) or to enforce logical constraints (see e.g. (14) and (20)).

In general, the MPC iteration (33) will be infeasible whenever there is an issue of scarcity of resources within the building. For example, (2) and (3) are violated in case of overload, (8) and (9) in case there is not sufficient time to recharge the vehicle or the ESS, (27) in case there is not enough time and/or energy available to heat the building satisfying the minimum required temperature, etc. In this respect, some considerations have to be made: first of all, proper dimensioning of the thermal and electrical systems should make the occurrence of overloads very rare. Secondly, notice that, event assuming the building to be in a potential overloading condition (e.g. cold winter day with numerous demanding load request, such as many concurrent EV recharging requests), the proposed method makes sure that a feasible solution is returned, provided that it exists (this is because it is based on constrained optimization, i.e., it directly embeds the constraints which may lead to infeasibility).

When instead constraints cannot be satisfied, different strategies can be adopted, such as: selective shedding of loads, possibly based on a shedding priority list (see e.g. [50]), relaxation of (soft) constraints (such as the ones on temperature bounds), as discussed, e.g., in [51], Section 1.2.5. 
By adopting the latter strategy for example, the soft constraints will be violated every time there are no sufficient resources to satisfy them, and the violation will be the least necessary to keep the problem feasible.

A second question concerns recursive feasibility, i.e., the assurance that, if the MPC problem is feasible at a given time, then it will remain feasible for all the successive iterations. Also in this case, from a theoretical point of view, the presented problem cannot be guaranteed to be recursive feasible. To see this, consider the generic MPC iteration at a given time $k$ and assume it is feasible (feasibility depending on the value of all the problem parameters and inputs in the prediction window $[k, k+N-1])$. The feasibility or infeasibility of the following MPC iteration at $k+1$ will depend on the value of the problem inputs at time $k+N$ (i.e., the new time interval entering the prediction window), which could be such that the iteration is infeasible. As explained e.g. in [51], recursive feasibility could be ensured by proper design of additional terminal constraints and terminal cost terms (denoted in the following as "terminal ingredients"), and with the inclusion of assumptions on the exogenous signals (i.e., the external temperature $T^{e x t}$, the renewable power profile $P^{p v}$, the power profiles $P^{p l}, P^{e v}$ associated to the user requests, etc.). However, on one hand, this could be a very complex task and, on the other hand, ensuring recursive feasibility would be obtained at the expense of a decrease of the feasibility region of the problem (due to the inclusion of the terminal ingredients, which could over constraint the problem). For this reason, the implementation of dedicated infeasibility management strategies (as the two ones outlined in the above), appears a practical and convenient solution.

3) MPC Stability: A full and comprehensive analysis of the stability properties of the presented control system is beyond the scope of the paper and constitutes future works. The analysis is made complex in particular by the presence of Boolean variables and of the exogenous signals. In the following, we highlight the theoretical properties that differentiate the present problem from standard MPC (for which many stability results are known, see e.g. [51]), and we sketch a stability proof in a simplified yet meaningful scenario, with punctual references to the specialised literature of interest. To start, we denote with $l\left(x_{i}, u_{i}\right)$ the stage cost in (1) (i.e., $V=\sum_{i=k}^{k+N-1} l\left(x_{i}, u_{i}\right)$ ). In this section, $x_{i}$ denotes the vector grouping all the state variables of the system at $i$ (the state of charge variables $S O C^{e s s}$ and all the controlled temperature variables, $T^{a i r}$, $T^{\text {floor }}, T^{\text {wall }}, T^{\text {boiler }}$, in the building units), while $u_{i}$ denotes the control variables, which ultimately reduces to $P_{i}^{p o c}$, as explained in the following. We consider a setting in which all the exogenous signals are constant, because, in the opposite case, the state trajectories would depend on the characteristics of those signals, and the stability problem would be properly framed as a problem of stability of trajectories, rather than stability of states (an even more complex stability question, that could be tackled in future works based on the research on periodic MPC, see e.g. [52]). Notice further that no Boolean variables appear in the objective function (1), which explicitly depends on the control variable $P^{p o c}$ only (which in turn depends only on $P_{i}^{h p}$, being all the remaining variables in (5) assumed constant). Therefore, we reduce to the analysis of a simplified problem which includes the objective function (1), and the dynamics equations (23), (26), (27), (28), (29), which are collectively denoted generically as $x_{i+1}=f\left(x_{i}, u_{i}\right)$, plus all the remaining constraints rewritten in general form as $x_{i} \in \mathrm{X}$ and $u_{i} \in \mathrm{U}$, with $\mathrm{X}$ and $\mathrm{U}$ compact sets. A first aspect which differentiates the formulation in this paper from standard MPC is as follows. If we denote with $\left(x^{s}, u^{s}\right)$ the best feasible steady-state control-input pair (i.e. the statecontrol pair that minimizes $l$, such that $x^{s}=f\left(x^{s}, u^{s}\right)$, $x^{s} \in \mathrm{X}$ and $\left.u^{s} \in \mathrm{U}\right)$, we have that $l(x, u) \leq l\left(x^{s}, u^{s}\right)$ for some feasible $(x, u)$ not corresponding to any equilibrium pair (i.e. the best feasible steady-state control-input pair is not in general the minimizer of the stage cost function, and there are other feasible, non-equilibrium input-state couples with smaller associated stage cost). This fact, which is the characterizing aspect of economic MPC [53], complicates the stability study, since the objective function cannot be directly used as Lyapunov function for the stability proof [53]. In addition, no specific terminal ingredients are introduced in the formulation, in order not to reduce the feasibility region of the algorithm, as discussed in the previous subsection, and this further complicates the stability analysis (a typical terminal constraint considered which ensures stability under mild conditions is $x_{N}-x^{s}=0$, i.e., to require the state at the end of the control window to coincide with the leastcost equilibrium state, $x^{s}$ ). A theoretical result present in literature and of interest for the present case is illustrated in [54], Theorem 4.1, on the practical stability of EMPC without terminal ingredients. The theorem states that, under certain assumptions, outlined below, the EMPC scheme is such that the equilibrium $x^{s}$ is practically asymptotically stable (see [54], Definition 4.1), that is, the state trajectories converge to a neighbourhood of $x^{s}$. The same theorem proves that the neighbourhood shrinks to $x^{s}$ as $N \rightarrow \infty$ (incidentally, the same theory also proves recursive feasibility of the EMPC control - here addressed, we remind, in a simplified setting). The key assumptions needed to establish the above result are: i) a strict dissipativity of the MPC iteration, ii) exponential reachibility of $x^{s}$, and iii) an additional $n_{x}$-step reachibility condition of the Jacobian linearization of system $f(x, u)$ (with $n_{x}$ the dimension of $x$ ). These three assumptions are defined in details in [54], Definition 3.2, Assumption 4.2 and Assumption 4.3 , respectively. Given the system at study, assumptions ii) and iii) are satisfied provided that the system is correctly dimensioned (e.g., so that it is physically possible to steer the state to $x^{s}$, as requested by ii)), while Proposition 4.3 in [55] presents mild conditions (the presence of a Slater condition) under which assumption i) is satisfied, in a problem setting to which the case discussed in this paper can be reduced (control of linear systems and a linear-quadratic stage cost).

As explained above, in the full, complex scenario, the controller will have to find an optimal trade-off between its objectives, and the resulting feasible operating point (i.e., the state trajectories) will be in general time-varying, according to the characteristics of the exogenous signals. 


\section{VALIDATION}

In this section, the building EMS is validated via simulation. The section discusses the simulation cases study, the implementation of the algorithm and a set of validation simulations.

\section{A. Implementation of the algorithm}

The overall MPC problem presented in this paper has been coded using the Julia programming language (https://julialang.org/) [56], version 0.7. The quadratic optimization problem constituting the MPC iteration has been modeled using the Julia library JuMP [57] and solved with Gurobi (http://www.gurobi.com/). The simulations have been performed on a Window 10 machine, 64 bit, equipped with an Intel I7-5500U CPU $2.40 \mathrm{GHz}$ and 8 GB RAM.

\section{B. Case study and simulation setup}

A real case study of a smart building under construction in Italy, is considered. The architecture of the micro grid is an innovative fully electric system. The building is completely gas free as all systems are electrically supplied. The suggested micro grid consists of an innovative electric-power system characterized by the following: 1) a single point of connection to the grid; 2) a low-voltage main switch board (MSB) to supply both the common services (heating, elevators, etc.) and the units; 3) a common PV installed on the roof of the building and connected to the MSB; 4) a distribution power system from the MSB for all common technical building systems (TBS); 5) a feeder distribution from the MSB supplying each unit by an independent feeder in a radial scheme; 6) a common ESS with a capacity of $100 \mathrm{kWh}$ and a maximum power of $10 \mathrm{~kW}$ is connected to the micro grid. The building is composed of 20 units, with a peak demand of $50 \mathrm{~kW}$. The micro grid supplies: the units of the building (lighting and sockets), the smart loads of the units (one dishwasher and one washing machine for each unit), the central heating system consisting in a heat pump, a charging station for 5 PEVs. The main simulation parameters are as follows: 5 PEV recharging requests and 31 load requests are considered during the day in order to create a realistic power demand profile and to demonstrate the ability of the controller to manage loads while satisfying user preferences. Heating (HVAC) and domestic hot water (DHW) systems are composed of: 1) a central heating and cooling station with electricity-driven heat pumps; 2) heating, cooling, and DHW distribution system for each unit; 3) a central thermal storage; 4) a metering satellite (SC) for each unit with meters. Heating energy demand of the building is limited by the thick insulation of the envelope. The sampling time $\mathcal{T}$ is equal to 5 minutes. The micro grid requires a building automation control system to improve its effectiveness. The technical building systems are flanked by a building automation control system with a main server able to host a supervisory system.

In the following, to simulate realistic operation conditions and to account for disturbances and modeling uncertainties, a zero-mean Gaussian noise is added to the state feedback taken by the controller at each time, i.e., in particular to the various

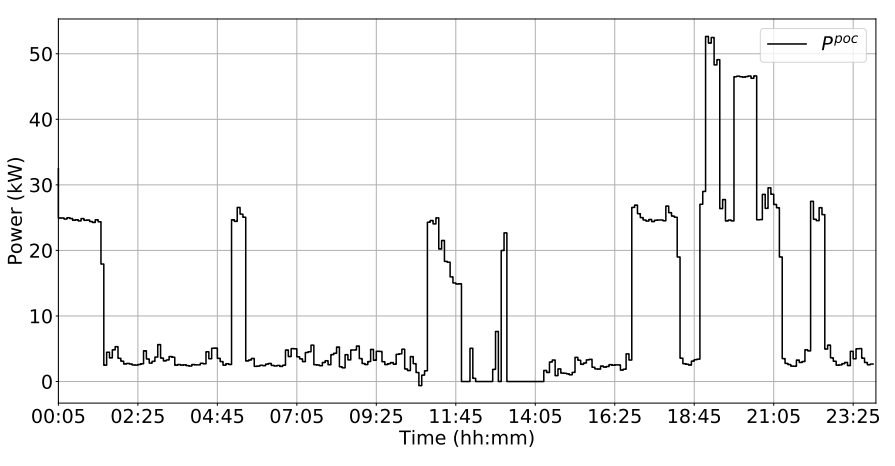

Fig. 3. Simulation 1: power flow at point of connection with the grid.

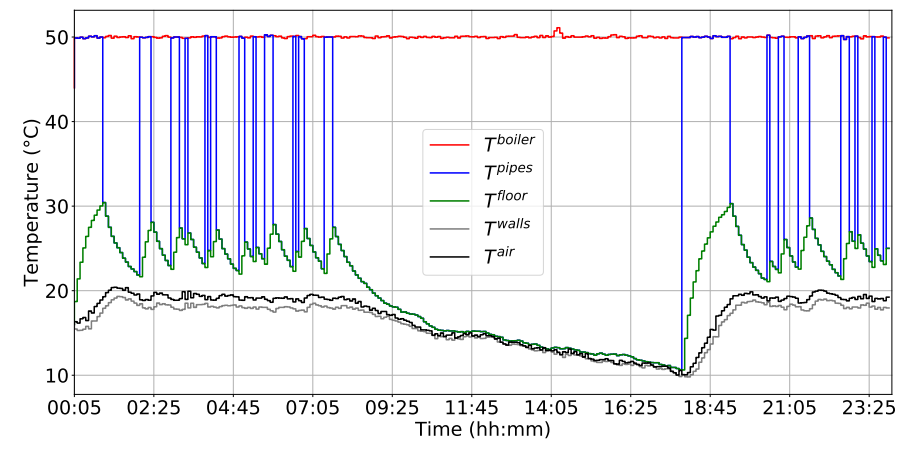

Fig. 4. Simulation 1: temperatures in building unit 1.

temperature measurements taken (i.e., to the feedback of the units' temperature and boiler temperature). The variance of the added Gaussian noise is $0,19{ }^{\circ} \mathrm{C}$, meaning that in the $99 \%$ of the cases, the added noise will be less than $0,5{ }^{\circ} \mathrm{C}$.

\section{Simulation 1: Baseline scenario with standard control}

The first simulation considers standard control of the building, in order to provide a baseline and a benchmark case for evaluating, in the following simulations, the increase in performance assured by the proposed control scheme. The control assumed here is: i) constant charging of the vehicles, at the maximum power allowed at the time of charging; ii) plannable loads are started at the first feasible time; iii) on/off control of the temperature (i.e., heating at maximum level when the temperature is outside of a band of 1 degree around the reference temperature); iv) the storage is always off.

Figure 3 reports the resulting power flow at the point of connection with the grid. As it could be expected, the power flow is not smooth and exhibits significant variations when plannable loads or PEV charging start (in particular, the $20 \mathrm{~kW}$ spikes in the figure correspond to the uncontrolled PEV charging processes). Figure 4 reports the temperature profile in the first building unit (temperature shows a similar pattern in the other units). The pattern of on-off control is recognised. Table I reports a more quantitative evaluation of the simulation for one sample unit (unit 1), in terms of: i) the average temperature error in the unit $\left(\operatorname{avg}\left(\operatorname{err}_{T^{a i r}}\right)\right)$ and ii) the standard deviation of the temperature error $\left(s t d\left(\operatorname{err}_{T^{a i r}}\right)\right)$, both as a proxy of the achieved user comfort; iii) the standard deviation of the power flow at the point of connection $\left(s t d\left(P^{p o c}\right)\right.$, as a measure 
TABLE I

KEY PERFORMANCE INDICATORSS (KPIS) OF SIMULATION 1 (BASELINE SCENARIO).

\begin{tabular}{ccccc}
\hline $\operatorname{avg}\left(\operatorname{err}_{T^{a i r}}\right)$ & $\operatorname{std}\left(e r r_{T^{a i r}}\right)$ & $s t d\left(P^{p o c}\right)$ & $\int P^{p o c}$ & $\max \left(P^{p o c}\right)$ \\
\hline \hline $1.4{ }^{\circ} \mathrm{C}$ & $1.9^{\circ} \mathrm{C}$ & $12.34 \mathrm{~kW}$ & $248.17 \mathrm{kWh}$ & $52.64 \mathrm{~kW}$ \\
\hline
\end{tabular}

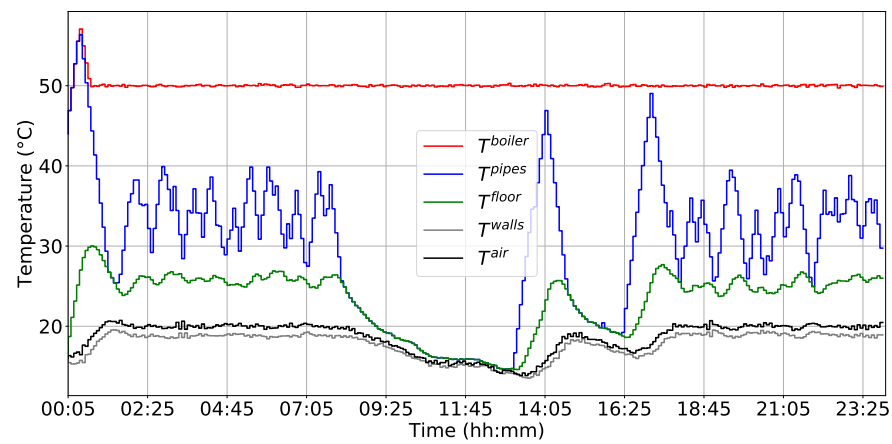

Fig. 5. Simulation 2: temperatures in building unit 1 .

of the smoothness of the flow); iv) the total energy flowing at the point of connection with the grid $\left(\int P^{p o c}\right.$, the lower, the better); and v) the electrical power peak $\left(\max \left(P^{p o c}\right)\right.$, the lower, the better). After a baseline scenario has been established, in the following sections the performance of the proposed controller is showcased under different scenarios of growing complexity and completeness.

\section{Simulation 2: Pure thermal management}

We show in this simulation the performance of the controller when its only goal is to best manage thermal loads in the building and satisfy the associated user preferences. To this end, $a_{1}=a_{4}=a_{5}=0$ is selected in (1) (the other weights are set to 1). PEVs and plannable loads are still started manually, as in the previous simulation. Similarly, the ESS is not activated. This is done in order to show how the controller performs when the only load to manage is the thermal one.

Figure 5 shows the evolution of the temperature in unit 1. It shows that accurate tracking of the reference temperature is achieved. The power profile at the point of connection is not shown here as it is almost the same as the one in Simulation 1. Table II reports the KPIs for this simulation (the peak power reduction and the cost saving compared to Simulation 1 are also reported in this case). Temperature tracking performance is excellent, while it is not surprising that the two other KPIs have values comparable with those in Simulation 1.

\section{E. Simulation 3: Joint thermal/electrical load management}

In this section all the terms in the objective function are enabled (all weights in (1) are set to one).

Figure 7 reports the profile of the power flow between the building and the grid. By comparing it with Fig. 3 it is seen that

TABLE II

KPIS OF SIMULATION 2 (PURE TEMPERATURE CONTROL).

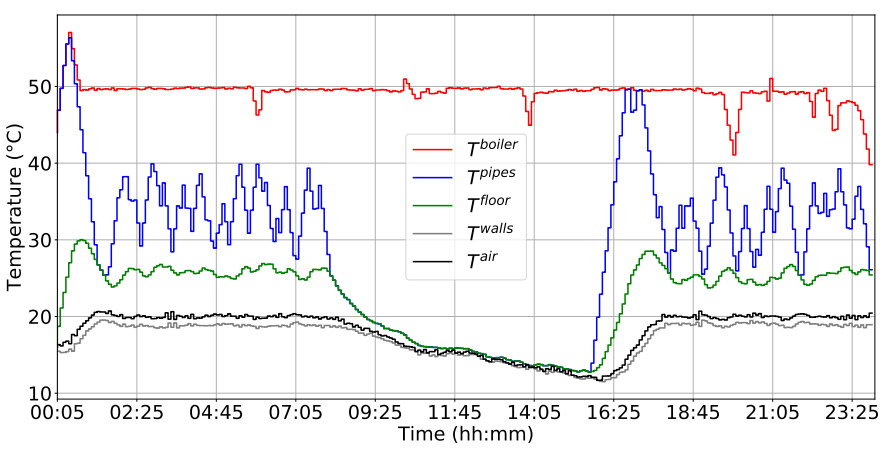

Fig. 6. Simulation 3: temperatures in building unit 1 .

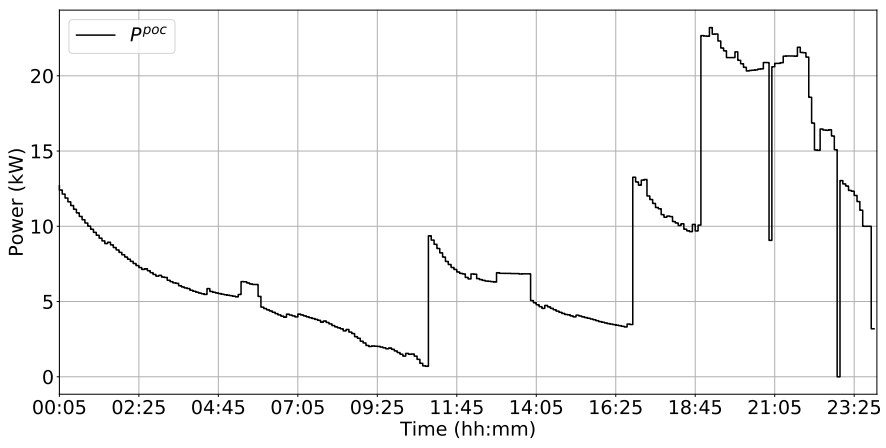

Fig. 7. Simulation 3: power flow at point of connection with the grid.

a much smoother flow is achieved. Figure 6 and 8 report the temperature profiles in unit 1 and in unit 11 (two representative units in the building). The two units have different occupancy times, and the controller ensures excellent performances in both cases. Notice how the controller manages the thermal storage property of the building by "pre-heating" the structures in advance of the hours when temperature tracking has to be ensured. The above qualitative behaviour of the controller is confirmed by the quantitative KPIs resulting from the simulation, reported in table III.

TABLE III

KPIS OF SIMULATION 3 (FULL CONTROL).

\begin{tabular}{ccccccc}
\hline \hline $\operatorname{avg}\left(e r r_{T_{\text {air }}}\right.$ & $s t d\left(\operatorname{err} T_{\text {air }}\right)$ & $s t d\left(P^{\text {poc }}\right)$ & $\int P^{\text {poc }}$ & $\max \left(P^{\text {poc }}\right)$ & Peak Reduction & Saving \\
\hline $0.36^{\circ} \mathrm{C}$ & $0.7^{\circ} \mathrm{C}$ & $6.25 \mathrm{~kW}$ & $205.9 \mathrm{kWh}$ & $23.2 \mathrm{~kW}$ & $-55,93 \%$ & $-19,35 \%$ \\
\hline
\end{tabular}

It is seen that the controller significantly smooths the power

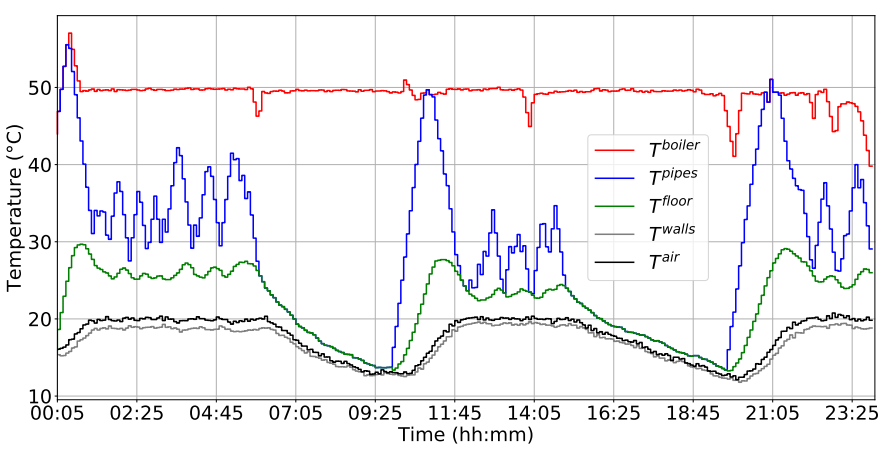

Fig. 8. Simulation 3: temperatures in building unit 11. 


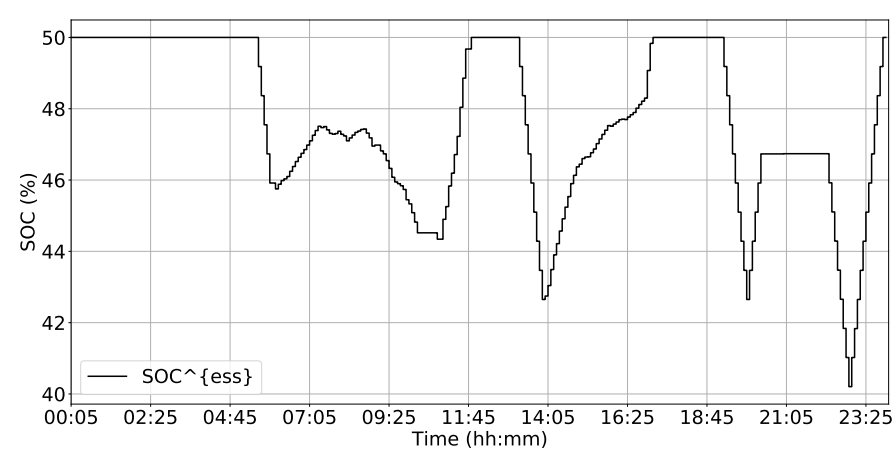

Fig. 9. Simulation 3: evolution of the ESS state of charge.

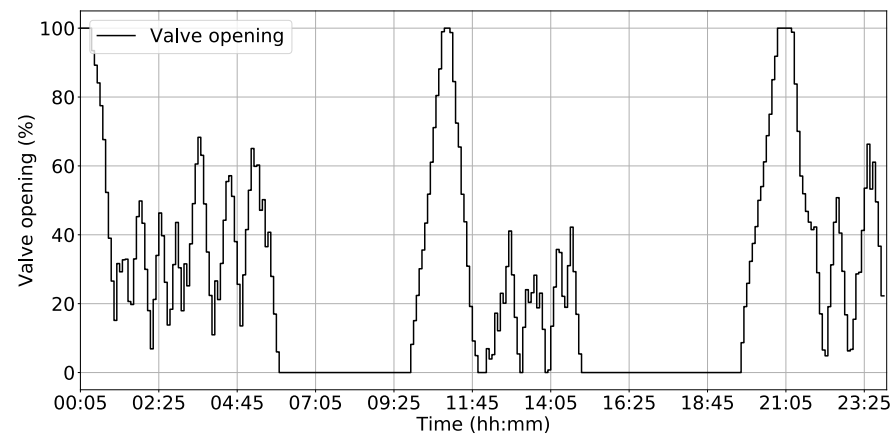

Fig. 10. Simulation 3: Opening position of the hot water valve in unit 1.

flow at the point of connection with the grid, ensures the same temperature control performance as in the previous case and achieves a significant reduction of the energy cost. Moreover, a halving of the peak power withdrawn from the grid is achieved. This is done by intelligent management of the ESS, of the recharging process of the PEVs, of the thermal storage of the building and of the start time of the plannable appliances. Also, the amount of energy flowing at the point of connection is significantly reduced as well, thanks to the self consumption of locally produced PV power.

To showcase how the controller manages loads' flexibility, Fig. 9 reports the evolution of the SOC of the ESS. It is seen how the ESS is controlled to contribute to optimizing the building's energy management, while keeping the evolution of the SOC close to the reference value (50\%), so to ensure adequate control margins.

Finally, we report in Fig. 10 the hot water flow valve opening position for unit 1 , to show that the proposed control does not introduce undesired chattering behaviour on the actuators. Rate of change constraints and or specific terms in the objective function (to penalise excessive variations in the actuators' inputs) can be added in general to avoid that the resulting control cause high fatigue on the actuators.

\section{F. Reaction to demand side management signals}

Buildings will be in the near future one of the main source of flexibility in the local flexibility electricity markets. Aggregators will pool coalitions of buildings to create a critical mass sufficient to participate in local markets. Price and volume signals will be exchanged between the aggregator and the buildings as a tool to explore the flexibility available at the building side to modify the demand profile.

The proposed algorithm is naturally capable to re-program the management of the building's load in order to react to a price signal from the aggregator, that is, a variation of the price of the energy in a given time interval. Volume signals can be considered as well, that is, requests of reduction/increase of the power flow in a given temporal interval.

\section{G. "Gentle service degradation"}

The proposed controller can cover emergency scenarios and for example ensure quality of experience preservation or gentle service degradation in presence of disruption events, like the temporal loss of the main supply from the grid. The proposed controller is capable to react to notification of the interruption of the power supply from the grid. The controller is capable of reacting as well to abrupt interruption of main power supply, however it would have naturally reduced control margin.

\section{$H$. Tuning of the controller parameters}

The quantitative performance and the behaviour of the building will vary depending on the selected parameters of the controller, principally the 5 weights in the objective function. The above simulations were meant to show the performance of the controller in different scenarios and were carried out with an empirical selection of the weights of the objective function. One way to tune the proposer controller is to test the performance in typical operating conditions of the building and varying the weights of the objective function. The KPIs examined in the above sections will form a Pareto frontier. By having these charts the building manager would be able to select the weights depending on his/her optimization goals and priorities (e.g. cost optimization or power smoothing).

\section{CONCLUSION}

This paper has presented a model predictive control based energy management algorithm for managing heating and electricity loads in a smart building. The managed loads include a heat pump, an electricity storage, electric vehicles and flexible loads. The control objective has been to minimize costs of energy consumption while ensure that the technical constraints of the system are met and the user preference are satisfied, in terms of thermal comfort and execution of loads according to the schedule set by the user (notably including control of electric vehicles). Explicative simulations and KPIs have been presented to show the effectiveness of the controller in optimising resources in a complex and realistic scenario.

Future works will regard the integration of the proposed control scheme with additional control devices, such as air conditioners, and with the description of other relevant physical phenomena, such as radiant and convective loads. More extensive simulations will also be conducted, spacing over different seasonal conditions, in order to fully validate the approach and obtain a complete performance assessment before being validated on real equipment. 


\section{REFERENCES}

[1] Organisation for Economic Co-Operation and Development, Energy Technology Perspectives 2017: Catalysing Energy Technology Transformations. OECD, 2017. [Online]. Available: https://webstore.iea.org/energy-technology-perspectives-2017

[2] European Parliament, Council of the European Union, "Directive 2010/31/EU of the European Parliament and of the Council of 19 May 2010 on the energy performance of buildings," Official Journal of the European Union, vol. 153, p. 1335, 18.6.2010. [Online]. Available: http://data.europa.eu/eli/dir/2010/31/oj

[3] — "Directive (EU) 2018/844 of the European Parliament and of the Council of 30 May 2018 amending Directive 2010/31/EU on the energy performance of buildings and Directive 2012/27/EU on energy efficiency (Text with EEA relevance)," Official Journal of the European Union, vol. L 156, p. 7591, 19.6.2018. [Online]. Available: http://data.europa.eu/eli/dir/2018/844/oj

[4] B. Kouvaritakis and M. Cannon, "Model predictive control," Switzerland: Springer International Publishing, 2016.

[5] M. Beaudin and H. Zareipour, "Home energy management systems: A review of modelling and complexity," Renewable and Sustainable Energy Reviews, vol. 45, pp. $318-335,2015$.

[6] Z. Yang, M. Y. Chow, G. Hu, and Y. Zhang, "Guest editorial new trends of demand response in smart grids," IEEE Transactions on Industrial Informatics, vol. 11, no. 6, pp. 1505-1508, Dec 2015.

[7] H. Shareef, M. S. Ahmed, A. Mohamed, and E. A. Hassan, "Review on home energy management system considering demand responses, smart technologies, and intelligent controllers," IEEE Access, vol. 6, pp. 24 498-24 509, 2018.

[8] G. R. Newsham, B. J. Birt, and I. H. Rowlands, "A comparison of four methods to evaluate the effect of a utility residential air-conditioner load control program on peak electricity use," Energy Policy, vol. 39, no. 10, pp. $6376-6389,2011$, sustainability of biofuels.

[9] J. Widn, "Improved photovoltaic self-consumption with appliance scheduling in 200 single-family buildings," Applied Energy, vol. 126, no. Supplement C, pp. 199 - 212, 2014.

[10] G. R. Newsham and B. G. Bowker, "The effect of utility time-varying pricing and load control strategies on residential summer peak electricity use: A review," Energy Policy, vol. 38, no. 7, pp. 3289 - 3296, 2010, large-scale wind power in electricity markets with Regular Papers.

[11] J. Torriti, "The significance of occupancy steadiness in residential consumer response to time-of-use pricing: Evidence from a stochastic adjustment model," Utilities Policy, vol. 27, no. Supplement C, pp. 49 $-56,2013$.

[12] C. Bartusch and K. Alvehag, "Further exploring the potential of residential demand response programs in electricity distribution," Applied Energy, vol. 125, no. Supplement C, pp. 39 - 59, 2014.

[13] A. Kiessling, "Modellstadt mannheim (moma) - abschlussbericht: Beitrge van moma zur transformation des energiesystems fr nachhaltigkeit, beteiligung, regionalitt und verbundheid," Final Report, 2013.

[14] R. D'hulst, W. Labeeuw, B. Beusen, S. Claessens, G. Deconinck, and K. Vanthournout, "Demand response flexibility and flexibility potential of residential smart appliances: Experiences from large pilot test in belgium," Applied Energy, vol. 155, no. Supplement C, pp. $79-90$, 2015.

[15] E. Klaassen, C. Kobus, J. Frunt, and J. Slootweg, "Responsiveness of residential electricity demand to dynamic tariffs: Experiences from a large field test in the netherlands," Applied Energy, vol. 183, no. Supplement C, pp. 1065 - 1074, 2016.

[16] COMED, "The COMED residential real time pricing program," [Online]. Available: www.comed.com/Documents/., 2012.

[17] M. Muratori and G. Rizzoni, "Residential demand response: Dynamic energy management and time-varying electricity pricing," IEEE Transactions on Power Systems, vol. 31, no. 2, pp. 1108-1117, March 2016.

[18] M. Shakeri, M. Shayestegan, H. Abunima, S. S. Reza, M. Akhtaruzzaman, A. Alamoud, K. Sopian, and N. Amin, "An intelligent system architecture in home energy management systems (hems) for efficient demand response in smart grid," Energy and Buildings, vol. 138, no. Supplement C, pp. 154 - 164, 2017.

[19] A. Hawkes and M. Leach, "Modelling high level system design and unit commitment for a microgrid," Applied Energy, vol. 86, no. 7, pp. 1253 - 1265, 2009.

[20] A. Di Giorgio and L. Pimpinella, "An event driven smart home controller enabling consumer economic saving and automated demand side management," Applied Energy, vol. 96, pp. 92-103, 2012.
[21] S. Y. Derakhshandeh, A. S. Masoum, S. Deilami, M. A. S. Masoum, and M. E. H. Golshan, "Coordination of generation scheduling with pevs charging in industrial microgrids," IEEE Transactions on Power Systems, vol. 28, no. 3, pp. 3451-3461, Aug 2013.

[22] A. Di Giorgio and F. Liberati, "Near real time load shifting control for residential electricity prosumers under designed and market indexed pricing models," Applied Energy, vol. 128, pp. 119 - 132, 2014.

[23] J. Silvente, G. M. Kopanos, E. N. Pistikopoulos, and A. Espua, "A rolling horizon optimization framework for the simultaneous energy supply and demand planning in microgrids," Applied Energy, vol. 155, no. Supplement C, pp. $485-501,2015$.

[24] J. Silvente and L. G. Papageorgiou, "An MILP formulation for the optimal management of microgrids with task interruptions," Applied Energy, vol. 206, no. Supplement C, pp. 1131 - 1146, 2017.

[25] H. T. Roh and J. W. Lee, "Residential demand response scheduling with multiclass appliances in the smart grid," IEEE Transactions on Smart Grid, vol. 7, no. 1, pp. 94-104, Jan 2016.

[26] G. M. Kopanos and E. N. Pistikopoulos, "Reactive scheduling by a multiparametric programming rolling horizon framework: A case of a network of combined heat and power units," Industrial \& Engineering Chemistry Research, vol. 53, no. 11, pp. 4366-4386, 2014.

[27] F. Liberati and A. Di Giorgio, "Economic model predictive and feedback control of a smart grid prosumer node," Energies, vol. 11, no. 1, 2018 [Online]. Available: http://www.mdpi.com/1996-1073/11/1/48

[28] A. Parisio, E. Rikos, and L. Glielmo, "A model predictive control approach to microgrid operation optimization," IEEE Transactions on Control Systems Technology, vol. 22, no. 5, pp. 1813-1827, Sept 2014.

[29] N. Jayasekara and P. Wolfs, "A hybrid approach based on GA and direct search for periodic optimization of finely distributed storage," in Innovative Smart Grid Technologies Asia (ISGT), Nov. 2011, pp. 1-8.

[30] M. A. A. Pedrasa, T. D. Spooner, and I. F. MacGill, "Coordinated scheduling of residential distributed energy resources to optimize smart home energy services," IEEE Transactions on Smart Grid, vol. 1, no. 2, pp. 134-143, Sept 2010.

[31] L. D. Ha, S. Ploix, E. Zamai, and M. Jacomino, "Tabu search for the optimization of household energy consumption," in 2006 IEEE Int. Conference on Information Reuse Integration, Sept 2006, pp. 86-92.

[32] Y. Han, P. Young, and D. Zimmerle, "Microgrid generation units optimum dispatch for fuel consumption minimization," Journal of Ambient Intelligence and Humanized Computing, vol. 4, no. 6, pp. 685-701, 2013.

[33] Z. Yang, R. Wu, J. Yang, K. Long, and P. You, "Economical operation of microgrid with various devices via distributed optimization," IEEE Transactions on Smart Grid, vol. 7, no. 2, pp. 857-867, March 2016.

[34] C. Gamarra and J. M. Guerrero, "Computational optimization techniques applied to microgrids planning: A review," Renewable and Sustainable Energy Reviews, vol. 48, no. Supplement C, pp. 413 - 424, 2015.

[35] S. M. Nosratabadi, R.-A. Hooshmand, and E. Gholipour, "A comprehensive review on microgrid and virtual power plant concepts employed for distributed energy resources scheduling in power systems," Renewable and Sustainable Energy Reviews, vol. 67, no. Supplement C, pp. 341 363, 2017

[36] E. Matallanas, M. Castillo-Cagigal, A. Gutierrez, F. Monasterio-Huelin, E. Caamano-Martin, D. Masa, and J. Jimenez-Leube, "Neural network controller for active demand-side management with pv energy in the residential sector," Applied Energy, vol. 91, no. 1, pp. 90-97, 2012.

[37] D. O'Neill, M. Levorato, A. Goldsmith, and U. Mitra, "Residential demand response using reinforcement learning," in Smart Grid Coтmunications (SmartGridComm), First IEEE International Conference on, Oct. 2010, pp. 409-414.

[38] C. Yan, X. Xue, S. Wang, and B. Cui, "A novel air-conditioning system for proactive power demand response to smart grid," Energy Conversion and Management, vol. 102, pp. 239 - 246, 2015.

[39] G. M. Kopanos, M. C. Georgiadis, and E. N. Pistikopoulos, "Energy production planning of a network of micro combined heat and power generators," Applied Energy, vol. 102, no. Supplement C, pp. $1522-$ 1534, 2013, special Issue on Advances in sustainable biofuel production and use - XIX International Symposium on Alcohol Fuels - ISAF.

[40] P. Ivanova, A. Sauhats, and O. Linkevics, "District heating technologies: Is it chance for chp plants in variable and competitive operation conditions?" IEEE Transactions on Industry Applications, vol. 55, no. 1, pp. 35-42, Jan 2019.

[41] A. Pellegrino, V. R. M. Lo Verso, L. Blaso, A. Acquaviva, E. Patti, and A. Osello, "Lighting control and monitoring for energy efficiency: A case study focused on the interoperability of building management systems," IEEE Transactions on Industry Applications, vol. 52, no. 3 , pp. 2627-2637, May 2016. 
[42] C. Shao, Y. Ding, J. Wang, and Y. Song, "Modeling and integration of flexible demand in heat and electricity integrated energy system," IEEE Tran. on Sustainable Energy, vol. 9, no. 1, pp. 361-370, Jan 2018.

[43] S. H. Tindemans, V. Trovato, and G. Strbac, "Decentralized control of thermostatic loads for flexible demand response," IEEE Transactions on Control Systems Technology, vol. 23, no. 5, pp. 1685-1700, Sep. 2015.

[44] A. M. A. Ahmed, L. Mihet-Popa, C. Agert, Y. Zong, J. Bruna, and X. Xiao, "Potential energy flexibility for a hot-water based heating system in smart buildings via economic model predictive control," in 2017 International Symposium on Computer Science and Intelligent Controls (ISCSIC), Oct 2017, pp. 1-5.

[45] O. Kilkki, A. Alahivl, and I. Seilonen, "Optimized control of price-based demand response with electric storage space heating," IEEE Tran. on Industrial Informatics, vol. 11, no. 1, pp. 281-288, Feb 2015.

[46] S. Rastegarpour, M. Ghaemi, and L. Ferrarmi, "A predictive control strategy for energy management in buildings with radiant floors and thermal storage," in 2018 SICE International Symposium on Control Systems (SICE ISCS), March 2018, pp. 67-73.

[47] L. Martirano, E. Habib, A. Giuseppi, and A. D. Giorgio, "Nearly zero energy building model predictive control for efficient heating," in 2018 IEEE Industry Applications Society Annual Meeting, Sep. 2018, pp. 1-6.

[48] L. Martirano, E. Habib, G. Parise, G. Greco, M. Manganelli, F. Massarella, and L. Parise, "Demand side management in microgrids for load control in nearly zero energy buildings," IEEE Transactions on Industry Applications, vol. 53, no. 3, pp. 1769-1779, May 2017.

[49] L. Martirano, G. Parise, G. Greco, M. Manganelli, F. Massarella, M. Cianfrini, L. Parise, P. di Laura Frattura, and E. Habib, "Aggregation of users in a residential/commercial building managed by a building energy management system (bems)," IEEE Transactions on Industry Applications, vol. 55, no. 1, pp. 26-34, Jan 2019.

[50] V.-H. Bui, A. Hussain, H.-M. Kim, and Y.-H. Im, "Optimal energy management of building microgrid networks in islanded mode considering adjustable power and component outages," Energies, vol. 11, no. 9, p. 2351,2018

[51] J. B. Rawlings, D. Q. Mayne, and M. Diehl, Model Predictive Control: Theory, Computation, and Design. Nob Hill Publishing, 2017.

[52] J. Khler, M. A. Mller, and F. Allgwer, "On periodic dissipativity notions in economic model predictive control," IEEE Control Systems Letters, vol. 2, no. 3, pp. 501-506, July 2018.

[53] M. Diehl, R. Amrit, and J. B. Rawlings, "A lyapunov function for economic optimizing model predictive control," IEEE Transactions on Automatic Control, vol. 56, no. 3, pp. 703-707, March 2011.

[54] T. Faulwasser, L. Grüne, M. A. Müller et al., "Economic nonlinear model predictive control," Foundations and Trends ${ }^{\circledR}$ in Systems and Control, vol. 5, no. 1, pp. 1-98, 2018.

[55] T. Damm, L. Grne, M. Stieler, and K. Worthmann, "An exponential turnpike theorem for dissipative discrete time optimal control problems," SIAM Journal on Control and Optimization, vol. 52, no. 3, pp. 19351957, 2014. [Online]. Available: https://doi.org/10.1137/120888934

[56] J. Bezanson, A. Edelman, S. Karpinski, and V. Shah, "Julia: A fresh approach to numerical computing," SIAM Review, vol. 59, no. 1, pp. 65-98, 2017. [Online]. Available: https://doi.org/10.1137/141000671

[57] I. Dunning, J. Huchette, and M. Lubin, "Jump: A modeling language for mathematical optimization," SIAM Review, vol. 59, no. 2, pp. 295-320, 2017.

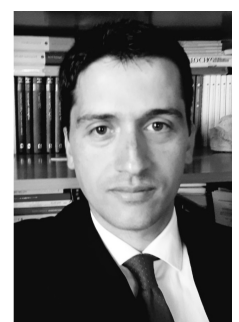

Francesco Liberati received his Ph.D. degree in systems engineering in 2015 from the University of Rome La Sapienza, Rome, Italy, where he is currently a research fellow. He is co-author of more than 30 technical publications on international journals, books and conference proceedings. He is/was involved in 9 European research projects, mainly dealing with ICT and Energy. His research interests include MPC and the application of system theory to recent problems in the fields of ICT and Energy.

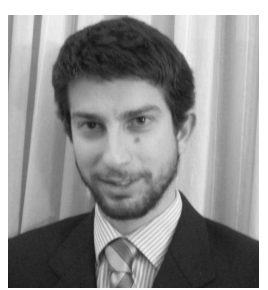

Alessandro Di Giorgio was born on May 15, 1980, in Rome, Italy. He received the Degree (cum laude) in physics in 2005, and the Ph.D. degree in systems engineering from the University of Rome La Sapienza, Italy, in 2010. He is currently an Assistant Professor of automatic control, working on original applications of control systems theory to smart grids, mainly developed in the context of European research projects. He is author of about 60 papers and book chapters on these topics.

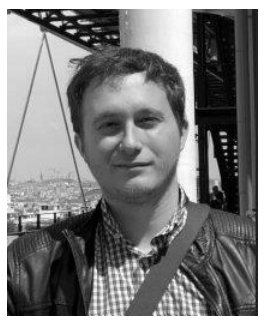

Alessandro Giuseppi was born in Rome, Italy, in 1992. He received from the University of Rome La Sapienza his B.Sc. degree in Computer and Automation Engineering in 2014 and his M.Sc. degree in Control Engineering in 2016, both summa cum laude. Currently, he is a Ph.D. Candidate in Automatica at the same university. He started his research on MPC in the context of the H2020 project ATENA, in which he studied Critical Infrastructures as Cyber-Physical Systems.

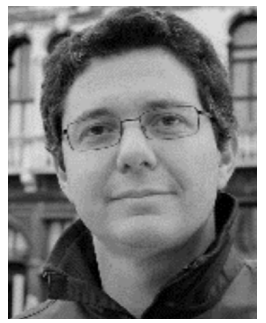

Antonio Pietrabissa received the M.Sc. degree in electronics engineering and the Ph.D. degree in systems engineering from the University of Rome La Sapienza, Rome, Italy, in 2000 and 2004, respectively. Since 2010, he has been Teaching Automatic Control and Process Automation with the University of Rome La Sapienza, where he is currently an Assistant Professor with the Department of Computer, Control, and Management Engineering Antonio Ruberti. Since 2000, he has been participated in about 20 EU and National Research Projects.

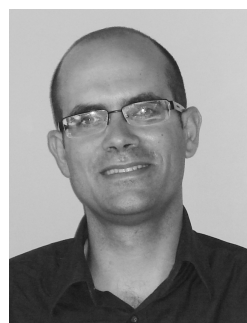

Emanuele Habib received M.S. degree in Mechanical Engineering in 2002 and Ph.D. in Applied Physics in 2007. In 2011, he joined the Department of Astronautics, Electrical and Energy Engineering of the Sapienza University of Rome where he is currently an Assistant Professor of Applied Physics. His main research fields are natural convection heat transfer, heat transfer through building envelope and interactions between HVAC systems, energy systems and buildings.

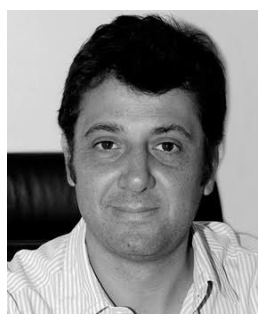

Luigi Martirano (StM98-M02-SM11) received the M.S. and Ph.D. degrees in Electrical Engineering in 1998 and 2003, respectively, from the Sapienza University, Rome Italy. In 2000, he joined the Department of Astronautics, Electrical and Energy Engineering of the Sapienza University of Rome where hes currently an Associate Professor of Electrical Power Systems and the coordinator of the $\mathrm{PhD}$ School of Science and Technology for Complex Systems. His main research fields are electric power systems, safety, building automation and energy systems. He's a co-founder of a startup about building automation systems applied for energy efficiency. 